\title{
Metodología Ciencia, Tecnología, Ingeniería y Matemáticas aplicada al Proceso de Enseñanza y Aprendizaje de las Ciencias Naturales
}

\author{
Science, Technology, Engineering and Mathematics Methodology applied to the \\ Teaching and Learning Process of Natural Sciences
}

Julia Viviana Argüello-Guevara ${ }^{1}$

\section{EDICIÓN. Resiliencia}

Recibido: 1/julio/2021

Aceptado: 1/octubre/2021

Publicado: 29/noviembre/2021

$\downarrow$ Páginas: $61-70$

4aís

${ }^{1}$ Colombia

IIIII Institución

${ }^{1}$ Universidad de Pamplona

Correo Eletrónico

1julia.arguello@unipamplona.edu.co

\section{(D) ORCID}

${ }^{1}$ https://orcid.org/0000-0001-8065-3762

\section{Citar así: Cf APA / IEEE}

Argüello-Guevara, J. (2021). Metodología Ciencia, Tecnología, Ingeniería y Matemáticas aplicada al Proceso de Enseñanza y Aprendizaje de las Ciencias Naturales. Revista Tecnológica-Educativa Docentes 2.0, 1(1), 61-70. https://doi.org/10.37843/rted.v1i1.256

J. Argüello-Guevara, "Metodología Ciencia, Tecnología, Ingeniería y Matemáticas aplicada al Proceso de Enseñanza y Aprendizaje de las Ciencias Naturales", RTED, vol. 1, n. ${ }^{\circ}$ 1, pp. 61-70, nov. 2021.

\section{Resumen}

La globalización y el surgimiento de la era tecnológica ha generado cambios en los oficios y profesiones más demandantes en el mundo entero, algunas han desaparecido y otras nuevas surgen, además las problemáticas de la sociedad requieren soluciones integrales y no parciales, según las áreas de conocimiento. El objetivo que orientó esta investigación se enfocó en analizar la incidencia de STEM en el proceso de enseñanza aprendizaje en el área de ciencias naturales de los estudiantes de quinto de básica primaria. Para tal efecto, se desarrolló una investigación acción de enfoque cualitativo en cuatro fases: planificación, acción, observación, reflexión. El aprendizaje en ciencias naturales bajo metodología STEM se delimitó a docentes y estudiantes de grado quinto primaria de la Institución Educativa Santo Ángel de carácter público en Bucaramanga, Colombia. Para tal fin, se eligió una muestra de 10 informantes claves, ampliamente conocedores, relacionados directamente con la investigación, distribuidos así, uno directivo docente, cuatro docentes de aula y cinco estudiantes. Como resultado al aplicar STEM como metodología de aprendizaje de ciencias naturales, los estudiantes lograron una buena adaptabilidad y aceptación al sistema de trabajo, así como una mejora significativa en su desempeño en la asignatura. En conclusión, el enfoque STEM mostró una amplia efectividad en el proceso de enseñanza aprendizaje de las ciencias naturales a través de actividades de tipo interdisciplinario.

Palabras clave: STEM, ciencias naturales, enseñanza, aprendizaje.

\section{Abstract}

Globalization and the emergence of the technological era have generated changes in the most demanding trades and professions worldwide. Some have disappeared, and other new ones arise; in addition, society's problems require integral and not partial solutions, according to the areas of knowledge. The objective that guided this research was to analyze the incidence of STEM in the teaching-learning process in the ??natural sciences of students in the fifth year of elementary school. For this purpose, action research with a qualitative approach was developed in four phases: planning, action, observation, reflection. Learning in natural sciences under STEM methodology was limited to fifth-grade teachers and students of the public Santo Ángel Educational Institution in Bucaramanga, Colombia. For this purpose, was chosen a sample of 10 key informants, widely knowledgeable, directly related to the research, distributed in this way, one teacher director, four classroom teachers, and five students. As a result, when applying STEM as a natural science learning methodology, the students achieved good adaptability and acceptance to the work system and a significant improvement in their performance in the subject. In conclusion, the STEM approach showed overall effectiveness in the teaching-learning process of natural sciences through interdisciplinary activities.

Keywords: STEM, natural sciences, teaching, learning. 


\section{Introducción}

La globalización y el surgimiento de la era tecnológica ha generado cambios en los oficios y profesiones más demandantes en el mundo entero, algunas han desaparecido y otras nuevas surgen, además las problemáticas de la sociedad requieren soluciones integrales y no parciales, según las áreas de conocimiento. Por lo anterior, desde la aplicación de la metodología STEM se pretende no solo generar más profesionales hombres y mujeres en las áreas STEM sino que sean capaces de brindar soluciones con una amplia visión y conocimiento. Como lo planteara Unesco "la educación en estas asignaturas puede proporcionar a quienes las estudian, los conocimientos, las habilidades, las actitudes y las conductas necesarias para crear sociedades inclusivas y sostenibles" (2017, p. 1)

Desde este planteamiento se destaca la exigüidad de profesionales con las habilidades que se requieren, no solo para afrontar las realidades del mundo sino para acercarse a un desarrollo pleno personal y profesional. De ahí lo esencial de comprometer a los estudiantes con el aprendizaje, como lo expresara, Kelley \& Knowles "se requiere de programas rigurosos que incluyan instrucción, evaluación, y también integrar la tecnología y la ingeniería en los planes de estudio de las ciencias y las matemáticas, así como promover la investigación científica y el proceso de diseño industrial" (2016, p.11). En otras palabras, se requiere definir el enfoque como lo manifestaran, Deirdre, Debby, Junie, \& Marnie "en los rincones de juego y de aprendizaje, los bloques y otros materiales abiertos ofrecen diferentes oportunidades" (2020, p. 37).

Con ello, los autores plantean de forma tácita el aporte del diseño como método de trabajo, de cierta forma este guarda similitud con el método científico en la búsqueda de verdad y soluciones, pero abriendo espacios al proceso de indagación para determinar cuál de ellas es la mejor, así como la posibilidad de optimizar y dinamizar procesos. Como lo expresaran, Borromeo, Mena, \& Mena "es esencial preparar a los futuros profesores de matemática, ciencia o tecnología para la integración de STEM, puesto que son el vehículo para implementar una formación acorde a las necesidades actuales" (2021, p. 45). De acuerdo con ello, las necesidades actuales hacen referencia al planteamiento de situaciones del mundo real en los contenidos temáticos, pues estos son complejos $\mathrm{y}$ requieren soluciones interdisciplinarias y a profundidad.

Desde de este contexto, analizar la incidencia del enfoque metodológico e interdisciplinar STEM en el proceso de enseñanza aprendizaje de las ciencias naturales como objetivo principal de esta investigación no solo contribuyo a optimizar practicas pedagógicas a mejorar el desempeño de los estudiantes, sino que permitió la proyección de las nuevas generaciones a un mejor estilo de vida, como lo explican Giraldo, Molina, \& Córdoba "los ciudadanos que no refuerzan estas áreas serán relegados de buenas oportunidades en la sociedad, ya que no poseerán las competencias necesarias para asumir determinadas tareas" (2018, p. 47). En otras palabras, que la implementación del enfoque STEM también permitiría ampliar las oportunidades profesionales y la mejora económica y sostenible del mundo entero.

\section{Metodología}

Para el desarrollo de esta investigación se aplicó el enfoque cualitativo que permitió desde sus parámetros abordar la incidencia de STEM en el proceso de enseñanza aprendizaje de las ciencias naturales de una forma humanista por su perspectiva social, en tal sentido, Serrano expresa: "el enfoque naturalista $o$ cualitativo surge como alternativa al paradigma racionalista, puesto que en las disciplinas de ámbito social existen diferentes problemáticas, cuestiones y restricciones que no se pueden explicar ni comprender en toda su extensión desde la metodología cuantitativa" (1994, p.26). Dicho de otro modo, para este caso de estudio de tipo social, de interacción y aprendizaje el 
paradigma cuantitativo no abarca todos los aspectos a valorar.

Bajo este enfoque investigativo se ajustan instrumentos de recolección de información para obtener datos del desempeño, actitudes y comportamiento de los estudiantes a través de un análisis riguroso de la información obtenida. Para tal fin, se elige una muestra de diez informantes claves, ampliamente conocedores, relacionados directamente con la investigación, distribuidos así, un directivo docente, cuatro docentes de aula y cinco estudiantes. A razón de ello, los instrumentos, entrevista a docentes, tabla de observación directa son elegidos y aplicados en la presente investigación, cabe acotar, fueron validados a través del mecanismo de validación por concepto de expertos.

La institución educativa Santo Ángel atiende a una población aproximada de 1500 estudiantes de estratos socioeconómicos bajos, en su mayoría, las familias dependen del trabajo informal, no han tenido acceso a educación superior y no cuentan con acceso a tecnologías de la información ni equipos de cómputo para facilitar el aprendizaje de los niños. Adicionalmente, el sector se caracteriza por sufrir flagelos sociales como drogadicción juvenil, delincuencia común y organizada, microtráfico, guerra entre pandillas, pobreza entre otros. Por ello la relevancia que toma el papel de la educación y las oportunidades que brinde a estos niños y jóvenes.

Ahora bien, congruentemente con el enfoque cualitativo, esta investigación se orienta bajo los parámetros del diseño de I-A (Investigación Acción) como ruta de trabajo, toda vez que I-A analiza acciones humanas, los problemas prácticos y cotidianos en las aulas de clase. Al respecto, Elliot plantea: "El propósito de la investigación-acción consiste en profundizar la comprensión del profesor (diagnóstico) de su problema. Por tanto, adopta una postura exploratoria frente a cualesquiera definiciones iniciales de su propia situación que el profesor pueda mantener" (2000, p.5). En otros términos, el autor propone que el docente debe partir de determinar las circunstancias actuales y preexistentes para de forma autocritica evaluar lo que se debe conservar y aquello que debe mejorar o replantear.

Con ello se hace evidente, el primer paso debe ser una actividad diagnóstica, pues esta permite ahondar y razonar acerca de las diferentes dificultades que se presentan en el aula entorno a un tema específico en este caso, al proceso de enseñanza aprendizaje de ciencias naturales en grado quinto primaria. Posteriormente, se realizó la investigación en cuatro fases principales en su orden: planificación, Acción, Observación, finalmente fase de Reflexión, como se observa en la siguiente Figura 1:

\section{Figura 1}

Rueda de Engranaje Cíclica

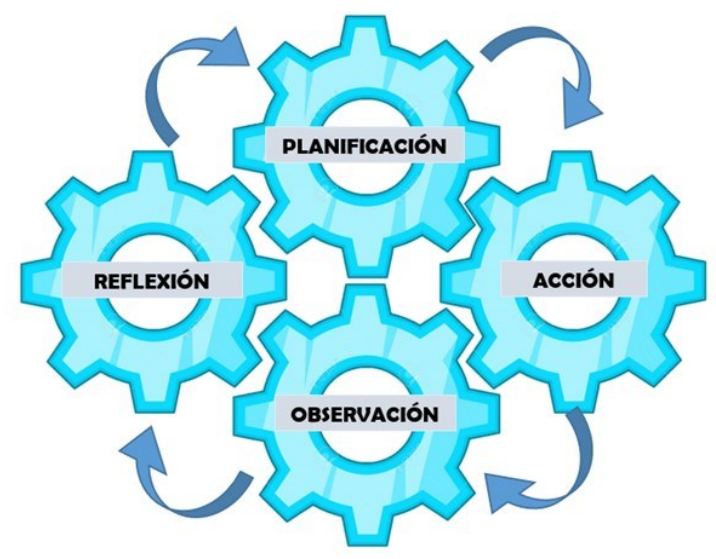

Nota. Proceso de elaboración de la investigación en cuatro fases, elaboración propia (2021).

Rueda de engranaje cíclica, muestra las diferentes etapas de las fases de investigación así como el flujo de información entre ellas. Sugiere la correlación positiva entre fases, sirviendo cada una de insumo para el desarrollo de la siguiente permitiendo un proceso consecuente. En el punto de partida es la planeación estructurada y sistemática, la puesta en práctica de las estrategias diseñadas, la observación de los efectos de las mimas y finalmente la reflexión sobre los resultados que lleve a las conclusiones definitivas, probablemente estás desencadenen en un nuevo planteamiento investigativo para permitir la evolución y mejora continua de los procesos. 
El registro de antecedentes permitió enmarcar la trayectoria de la metodología STEM, como se enriquece y se expande por el mundo. Al indagar al respecto, es visible cómo evoluciona $\mathrm{y}$ se afianza esta metodología, dejando un camino para rastrear, profundizar, aprender y por supuesto poner a prueba. Se encontraron numerosos estudios, con respecto a STEM y al proceso de enseñanza aprendizaje de las ciencias naturales, con mayor presencia en países como Estados Unidos, Finlandia, España, Alemania entre otros.

Por lo anterior y en relación con esta investigación, se destacan a nivel internacional, "Diseño de proyectos STEM a partir del currículo actual de educación primaria utilizando aprendizaje basado en problemas, aprendizaje cooperativo, Flipped classroom y robótica educativa" (Ruiz, 2007, p.197). Pues se muestro la posibilidad de engranar adecuadamente el currículo, la metodología ABP, la tecnología y el aprendizaje cooperativo a través de STEM.

A nivel nacional, en este caso en Colombia, se destacó la investigación denominada, "Metodologías didácticas para la enseñanza y aprendizaje de las ciencias naturales en zonas rurales del municipio de Obando - Valle del Cauca" (García, 2015, p. 59), pues concibe diferentes didácticas que invitan a un aprendizaje activo de las ciencias naturales incluso en zonas del país de difícil acceso lo que fue un gran aporte.

Por otra parte a nivel regional, se tuvo en cuenta el trabajo titulado, "Estrategia pedagógica de integración de la educación ambiental a las áreas de español, sociales y ciencias naturales para los estudiantes de grado $2^{\circ} 1$ del Instituto Comunitario Minca Sede C de Floridablanca: Una mirada transdisciplinar" (Gualteros, 2018, p. 136). Esta investigación aportó una experiencia positiva de integración de áreas como lo busca STEM en básica primaria.

Finalmente, a nivel local, "Miniproyectos: una estrategia metodológica basada en la enseñanza para la comprensión (EPC) en las ciencias naturales experimentales de escolares" (caicedo \& Acuña, 2015, p.26), dado que no solo demuestra un buen resultado de aplicación de las metodologías activas como forma de interacción en el aula sino que también muestra una manera experimental de aprender ciencias naturales, similar al objetivo STEM.

El análisis de los resultados se realizó a través de la técnica de la hermenéutica aplicada a la interpretación de los instrumentos de recolección de información, prueba diagnóstica, entrevista a docentes y observación directa.

\section{Resultados}

El momento histórico de la aplicación de esta investigación, valida aún más la búsqueda de la innovación en procesos de enseñanza aprendizaje, debido al confinamiento por la pandemia, la educación desde casa con mediación de la tecnología y estrategias innovadoras fue el reto de los agentes educativos en el mundo entero. Teniendo en cuenta lo anterior, la aplicación del enfoque STEM permitió desarrollar aprendizaje significativo a través de las metodologías activas, romper las barreras que aíslan las áreas del conocimiento y abarcar de manera más profunda e integral las temáticas, la motivación en los estudiantes fue notoria, encontrar que con una misma actividad estaban cubriendo sus compromisos escolares de varias asignaturas y de manera activa no memorística permitió obtener su atención y empeño en el desarrollo de las actividades que confluyeron en la mejora considerable de su desempeño académico en ciencias naturales.

\section{Metodología STEM}

La educación STEM "es un acercamiento interdisciplinario al aprendizaje que remueve las barreras tradicionales de las cuatro disciplinas (Ciencias, Tecnología, Ingeniería y Matemáticas) y las integra al mundo real con experiencias rigurosas $\mathrm{y}$ relevantes para los estudiantes" (Michael, Cary, \& Jo Anne, 2013, p. 50). Ese enfoque simpatizo entonces, por la transformación de las metodologías que aíslan por completo áreas de conocimiento evitando dar 
profundización al desarrollo de temáticas y ver el mundo me una forma más real.

Debe su nombre al acrónimo de las áreas fundamentales que desarrolla por sus nombres en inglés, Science (Ciencias) Tecnology (Tecnología) - Engineering (Ingeniería) - Mathematics (Matemáticas). Aparece por primera vez en los noventa cuando la NFS (National Science Fundation) hace una gran inversión para fortalecer las áreas impulsadas por STEM, posteriormente en la administración de Barack Obama es definida como política educativa pública. Desde allí, el STEM ha evolucionado y avanzado por el mundo. Se sustenta en tres grandes pilares, desarrollar una sociedad instruida en áreas STEM que permitan formar más profesionales en carreras que el mundo requiere, desarrollar nuevas generaciones con habilidades para el siglo XXI y propender por una sociedad basada en la innovación desde la ingeniería en la educación.

\section{La Ingeniería en la Educación}

Dentro de los aspectos que diferencian el enfoque STEM a otras innovaciones educativas, es la participación de la ingeniería como eje integrador entre las áreas establecidas por STEM. El objetivo principal de agregar ingeniería es dar un sentido de aprendizaje a partir de la solución de problemas y el aprendizaje basado en proyectos, que sin alejarse del todo de la rigurosidad de las ciencias y su método científico busca engranar de manera sistémica las áreas del conocimiento en busca de un mismo objetivo. "El método científico busca responder a una pregunta, mientras que el proceso de diseño busca encontrar soluciones y no cualquier solución, sino la mejor" (Espinosa, 2018, p.128). Con ello, se ha de entender por ejemplo, que con el método científico se válida una posible respuesta, mientras en el proceso de ingeniería desde el diseño, se busca una solución, se comprueba por demostración y se mejora por repetición.

Ahora bien, una de las más recurrentes preocupaciones de los docentes al asumir el reto de implementar STEM es el manejo conceptual que deben tener de ingeniería para desarrollar el enfoque metodológico con éxito, "Es poco probable que los docentes adopten la innovación o cambien su práctica docente a menos que tengan la suficiente confianza para implementarla. Por lo tanto, es necesario fortalecer la confianza de los profesores en el plan de estudios de ingeniería" (Lee \& Strobel, 2014, p. 163). Por ello, se hizo necesario la capacitación adecuada a los docentes para afianzar la confianza, brindar los conocimientos básicos del modelo y el aporte de la ingeniería en el proceso de enseñanza bajo el modelo STEM.

\section{Modelo de 5 pasos}

Desde la visión de la ingeniería en el desarrollo del pensamiento sistémico, STEM propone un modelo de actividades secuenciales de acuerdo con la complejidad y grado de los estudiantes, siendo el caso puntual de esta investigación el modelo de 5 pasos, correspondiente a los grados de 3ro a 8 vo. Estos modelos son los más utilizados para instrucción de la ingeniería en el aula, según lo expresa Espinosa:

Existen muchos modelos para representar el
proceso de diseño en ingeniería. Entre ellos se
encuentran los del tipo cíclico para representar
la reiteración. No siempre el problema indica
una reiteración, pero en esencia es un proceso
cíclico. También el modelo de forma cíclica
implica unos pasos fijos que aportan, cada uno,
a una forma de aprendizaje más profunda ( 2018 ,
p. 1).

Con ello el autor reitera la función de la ingeniería en el proceso de enseñanza aprendizaje desde el enfoque metodológico STEM, resaltando el objetivo de cada uno de los pasos del modelo de diseño en función de la instrucción y el desarrollo de competencias de los estudiantes y en el propender por el desarrollo de procesos cíclicos de mejora continua siempre que dé lugar.

En la Tabla 1 se muestra que con este modelo de 5 pasos una actividad STEM puede ser desarrollada con éxito, permitiendo el aprendizaje significativo y el desarrollo de contenidos curriculares de forma práctica y llamativa para los estudiantes. En cada uno de 
los pasos trabajados se potencializan competencias y habilidades que permitirán generaciones mejor instruidos en STEM y competitivos a nivel global.

Tabla 1

Modelo de Cinco Pasos

\begin{tabular}{|c|c|c|c|}
\hline \# & Paso & Descripción & $\begin{array}{l}\text { Habilidades } \\
\text { STEM que se } \\
\text { desarrollan }\end{array}$ \\
\hline 1 & $\begin{array}{l}\text { Hacer } \\
\text { preguntas }\end{array}$ & $\begin{array}{l}\text { Se presenta una situación o pregunta problema a los estudiantes, se } \\
\text { realiza una corta presentación de contexto del mismo para delimitar y } \\
\text { comprender su alcance, se permite a cada estudiante expresar sus } \\
\text { ideas. De esta forma el docente abre espacios para indagar los } \\
\text { presaberes y manejo que los estudiantes tienen del tema. }\end{array}$ & $\begin{array}{l}\text { Pensamiento } \\
\text { Critico } \\
\text { Investigación } \\
\text { Colaboración }\end{array}$ \\
\hline 2 & Imaginar & $\begin{array}{l}\text { Se abre el espacio a la creación, en donde una técnica de lluvia de } \\
\text { ideas por ejemplo, puede permitir que los estudiantes por mecanismos } \\
\text { de debates grupales, líderes en subgrupos entre otras, establezcan } \\
\text { ideas claras, argumentadas y las expongan en audiencia. }\end{array}$ & $\begin{array}{l}\text { Solución de } \\
\text { Problemas } \\
\text { Creatividad } \\
\text { Comunicación }\end{array}$ \\
\hline 3 & Planear & $\begin{array}{l}\text { Los estudiantes deben plasmar la solución que como grupo han } \\
\text { discutido o concertado a partir del diseño, para ello deben crear planos } \\
\text { o esquemas de muestren su solución con características específicas de } \\
\text { medidas, dimensiones, limitantes entre otras. Es recomendable en esta } \\
\text { etapa colocar limitantes similares a situaciones reales como el } \\
\text { presupuesto, los materiales, el tiempo entre otras. }\end{array}$ & $\begin{array}{l}\text { Solución de } \\
\text { Problemas } \\
\text { Creatividad } \\
\text { Comunicación } \\
\text { Colaboración }\end{array}$ \\
\hline 4 & Crear & $\begin{array}{l}\text { A partir de los planos deben iniciar la construcción de un prototipo } \\
\text { funcional que permita participación de todos los integrantes del grupo } \\
\text { de trabajo. Una de las estrategias recomendada para evitar estudiantes } \\
\text { rezagados en el proceso, es determinar el rol a desempeñar por cada } \\
\text { estudiante en la elaboración del prototipo, pueden usarse credenciales } \\
\text { o carné para identificar dicha labor a realizar y en cada sesión } \\
\text { reorganizar el rol de los estudiantes para permitir la experiencia desde } \\
\text { diversas ópticas. }\end{array}$ & $\begin{array}{l}\text { Pensamiento } \\
\text { Crítico } \\
\text { Solución de } \\
\text { Problemas } \\
\text { Creatividad } \\
\text { Comunicación } \\
\text { Colaboración } \\
\text { Liderazgo }\end{array}$ \\
\hline 5 & Mejorar & $\begin{array}{l}\text { Es de gran importancia en la experiencia de aprendizaje pues las } \\
\text { pruebas de funcionamiento del prototipo desarrollado deben dar como } \\
\text { resultado la solución esperada en el diseño inicial, de no ser así, el } \\
\text { trabajo debe revisarse, encontrar los errores e incluso en algunos casos } \\
\text { modificar el diseño hasta que cumpla su objetivo, sin importar cuantas } \\
\text { correcciones sean necesarias para alcanzar el objetivo. }\end{array}$ & $\begin{array}{l}\text { Pensamiento } \\
\text { Crítico } \\
\text { Solución de } \\
\text { Problemas } \\
\text { Creatividad } \\
\text { Comunicación } \\
\text { Colaboración }\end{array}$ \\
\hline
\end{tabular}

Nota. Se describen los modelos de una actividad STEM, elaboración propia (2021).

A partir de la tabla 1, es más explícito el quehacer en el aula del modelo de 5 pasos planteado por STEM en concordancia con la ingeniería en la educación, en ella a su vez se identifica un orden especifico de desarrollo de actividades en analogía a una secuencia didáctica que permita ejecutar una experiencia de aprendizaje práctica y significativa. Este modelo además responde a tres aspectos fundamentales que desde el proceso de diseño en la ingeniería, el primero de ellos, definir el problema, segundo, desarrollar soluciones y finalmente optimizar la solución.

\section{Interacción entre las Asignaturas STEM}

Definitivamente en Colombia y en muchos otros lugares del mundo existen bases muy arraigadas de modelos donde las áreas trabajan completamente aisladas, sustentados en la entrega de conceptos y memorización de una forma descontextualizada restando valor a los elementos teóricos por no enfocar su aplicación a las situaciones cotidianas que incluso pueden mejorar condiciones de vida de la comunidad educativa. 
De acuerdo con Espinosa "los problemas del mundo son eminentemente interdisciplinarios y por ello la combinación de las asignaturas permite mejores formas de solucionarlos. La integración de las asignaturas STEM es una forma efectiva para lograr la solución a problemas complejos y brindar al estudiante un aprendizaje más profundo" (2018, p.138). Con esto el autor afirma la importancia de comprender las situaciones problemas reales del mundo desde un todo, aprender a solucionar de forma fraccionada, nos lleva a dar alivios superficiales, a dejar brechas abiertas que pueden incluso complicar aún más el problema.

Con ello se hace necesario una visión más holística de situaciones problema e integración de las áreas, como lo plantea STEM, es una buena oportunidad para lograrlo. Sin embargo, debe existir claridad en la manera en que ellas se pueden integrar. STEM plantea diversas opciones de integración de áreas de acuerdo con los intereses o fines pertinentes, por ejemplo, silos, interconexión, coordinación, combinación, superposición, integración transdisciplinaria y para el caso de esta investigación, el modelo de asignatura líder, pues este engrana con en el objetivo que la sustenta, la búsqueda de mejorar el desempeño en el área de ciencias naturales de la población seleccionada.

El modelo de asignatura líder plantea que las áreas matemáticas y ciencias naturales al tener contenidos temáticos sólidos $\mathrm{y}$ robustos les permiten liderar procesos de aprendizaje integral, pues en ellas se sujetan y se pueden articular otras áreas, así pues, las ciencias naturales como área líder atrae a las otras para apoyar la temática trabajada, siendo la ingeniería la que establece las conexiones entre las áreas a través del proceso de diseño.

\section{Didáctica de las Ciencias Naturales}

Las ciencias naturales se han convertido en una disciplina de conocimiento esencial, pues se ha venido posicionando como promotora de cambio y eje de proyección de nuevas formas de comprender $y$ proteger nuestro planeta, teniendo en cuenta los retos ambientales, epidemiológicos, energéticos, farmacéuticos, todo lo que la ciencia permite desarrollar, es por ello, que la forma de enseñar ciencias naturales en el aula y la didáctica para tal fin deben ser planeadas $y$ estructuradas de forma responsable $y$ minuciosa.

Dentro del enfoque metodológico STEM, se concibe las ciencias naturales como un área completamente activa, que debe llevar al estudiante de lo teórico a lo práctico. Es por esto, que las metodologías activas engranan perfectamente en la propuesta STEM y lo planteado en los estándares de competencias establecidas por el MEN para el aprendizaje de las mismas. Partiendo de lo planteado por el MEN:

\begin{abstract}
A partir de dichas competencias generales, se establecen competencias más específicas, más aterrizadas a la acción en las aulas, a buscar los medios para alcanzar los objetivos, dentro de estas competencias específicas se encuentran, Identificar, Indagar, Explicar, Comunicar, trabajo en equipo, disponibilidad para tolerar la incertidumbre y aceptar la naturaleza provisional, propia de la exploración científica entre otras $(2004$, p. 7$)$.
\end{abstract}

En efecto, este postulado lista las competencias y habilidades propias que deben desarrollarse a través del estudio de las ciencias naturales, esto sumado a que constituyen políticas educativas de carácter nacional, revelan como STEM se adapta los currículos establecidos por la legislación y a su vez encaja en las necesidades de los estudiantes en su proceso de formación.

Con ello es claro qué se debe desarrollar desde el proceso de enseñanza aprendizaje de las ciencias naturales, en concordancia con los objetivos de STEM algunas de las metodologías activas que pueden dirigir actividades esta investigación son por ejemplo:

- ABP (Aprendizaje Basado en Problemas).

- Modelo de enseñanza de transmisión-recepción. 
- Modelo recepción significativa.

- Cambio conceptual.

- Modelo por investigación.

- Miniproyectos.

Cada una de ellas plantea un sin número de posibles actividades que permitan desarrollar un verdadero aprendizaje de ciencias naturales más desde el punto de vista del desarrollo de competencias y habilidades que desde el ejercicio de conceptualización mecánica y sistemática de conceptos. "Diagnosticar ideas y construir nuevos conocimientos, adquirir habilidades de rango cognitivo, promover actitudes positivas hacia la ciencia y actitudes científicas, acercar el ámbito científico a lo cotidiano y evaluar el conocimiento científico humano" (Ortega, 2007, p.1). En realidad, lograr este tipo trasformación de la educación, este acercamiento a la ciencia y a lo humano en los países de Latinoamérica permitiría un mejor desarrollo económico y social de nuestras naciones.

Tras la implementación de cinco actividades STEM en las que se cumplió con los parámetros de cada paso del diseño, donde la tecnología actúo como mediadora del proceso de aprendizaje y donde el centro fueron los estudiantes mientras que el docente asumió el rol de orientador facilitador del proceso, al finalizar la recolección de datos se encontró que:

1. Los docentes mostraron gran interés y curiosidad por el enfoque metodológico STEM por su visión e innovación integradora de las áreas.

2. Inicialmente los docentes manifestaron preocupación por la inclusión de la ingeniería como una asignatura adicional a evaluarse por la preparación que se debe tener para orientarla.

3. En el proceso de caracterización diagnóstica de los procesos de enseñanza aprendizaje de las ciencias naturales en la población de muestra, se evidencia un bajo desempeño académico en general, especial dificultad en solución de problemas contextualizados así como un arraigo a la forma tradicional de educación, memorística, de transcripción y repetición.

4. En la aplicación de actividades tipo STEM los estudiantes se mostraron, motivados, muy interesados y de cierta forma sorprendidos por el cambio de metodología de trabajo. Expresaron sentir compromiso al saber que la actividad vinculaba cumplimiento académico en varias asignaturas y muy receptivos frente a las indicaciones de las diferentes actividades.

5. Se desarrollaron a cabalidad las actividades propuestas, cabe mencionar que el proceso de actividades escolares en modalidad de alternancia por la pandemia por COVID-19, con llevo a realizar las actividades con pequeños grupos de estudiantes.

6. Al evaluar las temáticas trabajadas a través del enfoque metodológico STEM se evidencia un desempeño académico de los estudiantes ubicado principalmente en la escala alto y superior en escala de evaluación institucional y nacional.

7. Al evaluar a través de sustentación oral temáticas trabajadas a través de las actividades STEM, se evidencia un dominio significativo de los conceptos a través de la conceptualización.

\section{Conclusiones}

El enfoque metodológico STEM abre las puertas a nuevas oportunidades de innovación en el aprendizaje, a través de esta investigación se pudo comprobar no solo la gran acogida que tiene la metodología en docentes y estudiantes sino la efectividad en el proceso de enseñanza aprendizaje de las ciencias naturales en grado 5to primaria. La aplicación de los contenidos curriculares a problemáticas reales del entorno permitió una gran validación de los contenidos a los estudiantes, una apropiación de conceptos, 
realidades y el desarrollo de competencias acordes a las necesidades de la sociedad del siglo XXI.

Ahora bien, es importante reconocer que la mediación de la tecnología en el aprendizaje debe ser una constante en la educación y una oportunidad equitativa en todas las escuelas del mundo, censurar el uso del internet en el desarrollo de las clases debe cambiarse por enseñar a los niños y jóvenes a dar un buen uso de ella para su aprendizaje tal como lo propone STEM. Se pudo evidenciar en esta investigación, que el uso de las tecnologías de la información y la comunicación no solo fomenta la capacidad de indagación sino el pensamiento crítico ante lo que se encuentra en las redes y algo aún más importante, ser conscientes de la realidad que los rodea y como pueden mejorar su calidad de vida y la de su comunidad como lo busca STEM.

De acuerdo con lo planteado por Sandoval "cualquier propuesta que implique cambio y mejora utilizando las TIC se constituye como una innovación al proceso de enseñanza aprendizaje" (2020, p. 27). En otras palabras, que toda practica pedagógica que incluya mediación de la tecnología en su proceso, ya por sí misma supone novedades y modernización, así pues, las actividades STEM se constituyen en un cambio que propende por la mejora y por la integración cotidiana y constante de las TIC en la educación.

Finalmente, es válido recalcar que aplicar el enfoque metodológico STEM en los procesos de enseñanza aprendizaje en ciencias naturales, se da a esta importante área el toque activo propio de la misma, una asignatura tan empírica, no se debe seguir limitando a salón, marcador y tablero, pues una vez más queda demostrado, el aprender haciendo a través de experiencias significativas hace el aprendizaje más duradero y real. Por lo anterior cierro este apartado invitando a aprender más de STEM, de las oportunidades que esto abre a nuestra labor como educadores, a abrir puertas en las clases de ciencias naturales para ir hacia el exterior, para ir más a los laboratorios, a las salas de sistemas, a construir prototipos, a usar tecnología, a realizar clases conjuntas, a profundizar y a orientar a nuestros estudiantes a solucionar problemas reales y actuales de nuestra sociedad para que sigamos construyendo la expectativa de un mundo mejor.

\section{Referencias}

Borromeo, R., Mena, J., \& Mena, A. (2021). Fomento de la Educación STEM y la Modelización Matemática para profesores. Alemania: Kassel University Press. https://doi.org/doi:10.17170/kobra-202106174132

Caicedo, L., \& Acuña, M. (2015). Miniproyectos: Una Estrategia Metodológica Basada en la Enseñanza para la Comprensión (Epc). En Las Ciencias Naturales Experimentales De Escolares. Repositorio Institucional

$U N A B$. http://hdl.handle.net/20.500.12749/2285

Deirdre, E., Debby, M., Junie, B. K., \& Marnie, F. (2020). Juegos STEM en los Rincones de Aprendizaje (1 ra ed.). NARCEA S.A.

Elliot, J. (2000). La Investigación Acción en la Educación. Morata.

Espinosa, J. B. (2018). STEM Introducción a una nueva forma de enseñar y aprender. En J. B. Espinosa. STEM Education Colombia.

García, S. (2015). Metodologías didácticas para la enseñanza y aprendizaje de las ciencias naturales en zonas rurales del municipio de Obando - Valle del Cauca.

https://repositorio.unal.edu.co/bitstream/handle/unal /53550/Tesis\%20Sair.pdf?sequence $=1 \&$ isAllowed $=$ y

Giraldo, F., Molina, J., \& Córdoba, F. (2018). Desarrollo y Transformación Social desde Escenarios Educativos. Fondo Editorial ITM. https://books.google.com.co/books?id=swFgDwAA QBAJ\&pg=PA49\&dq=STEM+AUTORES\&hl=es419\&sa $=$ X\&ved $=2$ ahUKEwjP3eecy6DzAhV$\mathrm{SjABHb}$ mCSw4ChDoAXoECAQQAg\# $\mathrm{v}=$ onepage $\& \mathrm{q}=\mathrm{STEM} \% 20 \mathrm{AUTORES} \& \mathrm{f}=$ true

Gualteros, N. (2018). Estrategia pedagógica de integración de la educación ambiental a las áreas de español, sociales y ciencias naturales para los estudiantes de grado $2^{\circ} 1$ del Instituto Comunitario Minca Sede C de Floridablanca: Una mirada transdisciplinar. Universidad Santo Tomás. https://repository.usta.edu.co/bitstream/handle/1163 4/16311/2019nohoragualteros.pdf? sequence $=1 \&$ is A llowed $=\mathrm{y}$

Kelley, T., \& Knowles, J. (2016). Un marco conceptual para la educación STEM integrada. Revisa Internacional de Educación STEM. https://doi.org/10.1186/s40594-016-0046-z 


\section{Metodología Ciencia, Tecnología, Ingeniería y \\ Matemáticas aplicada al Proceso de Enseñanza y Aprendizaje de las Ciencias Naturales.}

Lee, J., \& Strobel, J. (2014). Ingeniería en entornos preuniversitarios: sintesis de investigación, políticas y prácticas.

MEN.

(2004).

Mineducación

https://www.mineducacion.gov.co/1759/articles-

81033_archivo_pdf.pdf

Michael, C., Cary, S., \& Jo Anne, V. (2013). STEM Lesson Essentials. En C. S. Michael Comer, STEM Lesson Essentials. Heinemann.

Ortega, F. J. (2007). EDUCREA. https://educrea.cl/modelosdidacticos-para-la-ensenanza-de-las-cienciasnaturales/

Ruiz, V. (2007). Diseño de proyectos STEM a partir del currículo actual de educación primaria utilizando aprendizaje basado en problemas, aprendizaje cooperativo, Flipped classroom y robótica educativa. Universidad CEU Cardenal Herrera. https://repositorioinstitucional.ceu.es/handle/10637/ 8739

Sandoval, C. H. (2020). La Educación en Tiempo del Covid19 Herramientas TIC: El Nuevo Rol Docente en el Fortalecimiento del Proceso Enseñanza Aprendizaje de las Prácticas Educativa Innovadoras. Revista Tecnológica-Educativa Docentes 2.0,9(2), 24-31. https://doi.org/10.37843/rted.v9i2.138

Serrano, G. P. (1994). Investigación Cualitativa. Retos e Interrogantes. La Muralla S.A.

Unesco. (2017). Descifrar el Código: La Educación de Niñas $y$ Mujeres en STEM. Unesco. https://books.google.com.co/books?id=8jOIDwAA QBAJ\&printsec $=$ frontcover\&dq $=$ educaci $\% \mathrm{C} 3 \% \mathrm{~B} 3 \mathrm{n}$ + STEM\&hl=es-

$419 \& \mathrm{sa}=\mathrm{X} \&$ redir_esc $=\mathrm{y} \# \mathrm{v}=$ onepage $\& \mathrm{q}=$ educaci $\%$ $\mathrm{C} 3 \% \mathrm{~B} 3 \mathrm{n} \% 20 \mathrm{STEM} \& \mathrm{f}=$ false 